\title{
Towards all-dielectric, polarization-independent optical cloaks
}

\author{
Andkjær, Jacob Anders; Mortensen, N. Asger; Sigmund, Ole
}

Published in:

Applied Physics Letters

Link to article, DOI:

$10.1063 / 1.3691835$

Publication date:

2012

Document Version

Publisher's PDF, also known as Version of record

Link back to DTU Orbit

Citation (APA):

Andkjær, J. A., Mortensen, N. A., \& Sigmund, O. (2012). Towards all-dielectric, polarization-independent optical cloaks. Applied Physics Letters, 100(10), Paper 101106. https://doi.org/10.1063/1.3691835

\section{General rights}

Copyright and moral rights for the publications made accessible in the public portal are retained by the authors and/or other copyright owners and it is a condition of accessing publications that users recognise and abide by the legal requirements associated with these rights.

- Users may download and print one copy of any publication from the public portal for the purpose of private study or research.

- You may not further distribute the material or use it for any profit-making activity or commercial gain

- You may freely distribute the URL identifying the publication in the public portal

If you believe that this document breaches copyright please contact us providing details, and we will remove access to the work immediately and investigate your claim. 


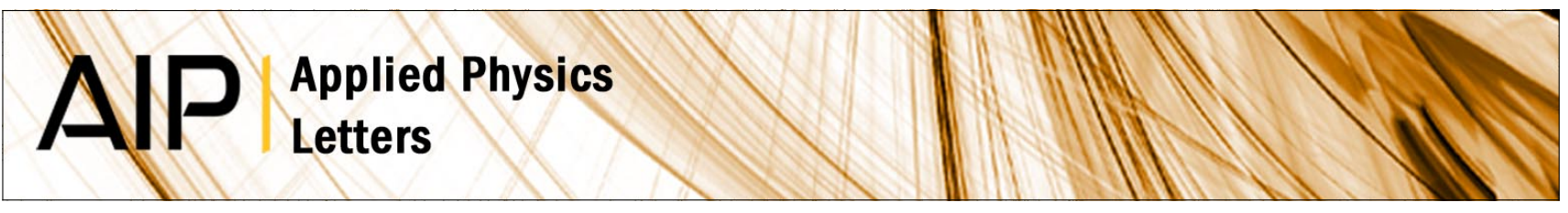

\section{Towards all-dielectric, polarization-independent optical cloaks}

Jacob Andkjær, N. Asger Mortensen, and Ole Sigmund

Citation: Appl. Phys. Lett. 100, 101106 (2012); doi: 10.1063/1.3691835

View online: http://dx.doi.org/10.1063/1.3691835

View Table of Contents: http://apl.aip.org/resource/1/APPLAB/v100/i10

Published by the American Institute of Physics.

\section{Related Articles}

Modal propagation and interaction in the smooth transition from a metal mushroom structure to a bed-of-nailstype wire medium

J. Appl. Phys. 111, 074308 (2012)

Light trapping beyond the $4 \mathrm{n} 2$ limit in thin waveguides

Appl. Phys. Lett. 100, 121121 (2012)

Effective permeability of a metamaterial: Against conventional wisdom

Appl. Phys. Lett. 100, 124103 (2012)

Liquid crystal based nonlinear fishnet metamaterials

Appl. Phys. Lett. 100, 121113 (2012)

Ultra-broadband microwave metamaterial absorber

Appl. Phys. Lett. 100, 103506 (2012)

\section{Additional information on Appl. Phys. Lett.}

Journal Homepage: http://apl.aip.org/

Journal Information: http://apl.aip.org/about/about_the_journal

Top downloads: http://apl.aip.org/features/most_downloaded

Information for Authors: http://apl.aip.org/authors

\section{ADVERTISEMENT}

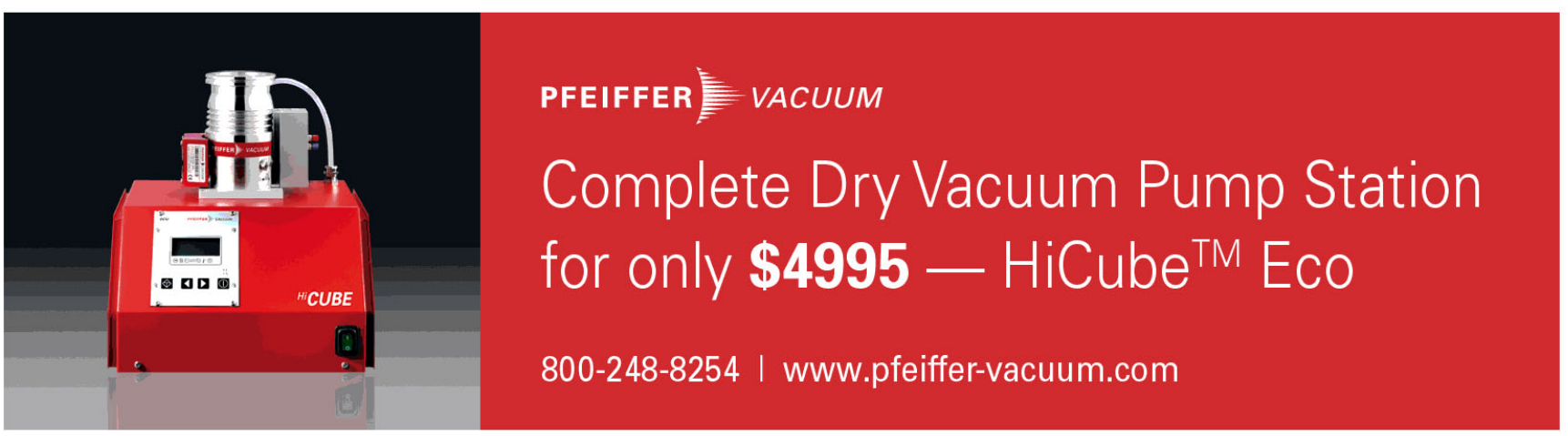




\title{
Towards all-dielectric, polarization-independent optical cloaks
}

\author{
Jacob Andkjær, ${ }^{1, \text { a) }}$ N. Asger Mortensen, ${ }^{2}$ and Ole Sigmund ${ }^{1}$ \\ ${ }^{1}$ Department of Mechanical Engineering, Technical University of Denmark, 2800 Kongens Lyngby, Denmark \\ ${ }^{2}$ Department of Photonics Engineering, Technical University of Denmark, 2800 Kongens Lyngby, Denmark
}

(Received 16 September 2011; accepted 12 February 2012; published online 6 March 2012)

Fully enclosing, all-dielectric cloaks working for both $E_{z}$ and $H_{z}$ polarizations simultaneously are presented in this letter. The cloaks are effective for two antiparallel angles of incidence, and the layout of standard dielectric material in the cloak is determined by topology optimization. Scattering from cylinder and cloak is reduced for an $H_{z}$-polarized wave compared to an $E_{z}$-polarized wave by taking advantage of the surface mode at the perfectly electric conducting boundary. Perhaps contrary to simple intuition, fully enclosed, all-dielectric, low-contrast cloaks cannot be designed effectively when distributing a material with lower permittivity than the background material. @ 2012 American Institute of Physics. [http://dx.doi.org/10.1063/1.3691835]

The layout of the material parameters for cloaks that manipulate electromagnetic waves to flow around an object without any scattering can conveniently be found by analytical transformation optics approaches. ${ }^{1,2}$ However, the required material parameters for fully enclosing or complementary medium-based ${ }^{3}$ cloaks are extreme in some regions of the cloak. Typical realizations ${ }^{4}$ rely on resonances having a narrow band and lossy response. Furthermore, subwavelength artificial structuring is challenging for optical frequencies. On the other hand, material parameters for alldielectric carpet cloaks ${ }^{5}$ need not to be extreme, thus circumventing the above challenges. Both two-dimensional (2D) and recently three-dimensional (3D) macroscopic alldielectric carpet cloaks have been demonstrated (cf. Refs. 6-9). Here, we address the more challenging problem of designing fully enclosing, all-dielectric cloaks.

Recent results ${ }^{10}$ show that gradient-based topology optimization ${ }^{11}$ can be used to find the permittivity distribution for a low-contrast, all-dielectric, fully enclosing optical cloak that hides an ideal metallic cylinder for up to 4 symmetrically distributed angles of incidence or for an incoming angular interval, of \pm 5 . The study was based on distributing material with a higher permittivity than the background material (free space) and illuminating by an $E_{z}$-polarized wave. In this letter, we present fully enclosing, all-dielectric cloaks effective for both $E_{z}$ and $H_{z}$ polarizations simultaneously. Furthermore, we study the cloaking effect of distributing material with either higher or lower permittivity than the background material. The optimized designs for the former case delay the waves in regions of higher permittivity than the background and, subsequently, phase match them to the waves outside. Intuitively, distributing dielectric material having a lower permittivity than the background material would result in cloaking performance on the same level or better; however, this is not the case as we will show.

The numerical setup is identical to the previous study. ${ }^{10}$ The computational domain is given in $2 \mathrm{D}$ using 3 concentric circles, see Fig. 1. The inner, middle, and outer domains represent the perfectly electric conducting (PEC) cylinder, the

${ }^{\text {a)} E l e c t r o n i c ~ m a i l: ~ j b a n @ m e k . d t u . d k . ~}$ cloak, and the surroundings, respectively. Assuming invariance of the electromagnetic properties along the cylinder axis, Maxwell's equations simplify to the scalar Helmholtz equation. The problem is solved using the finite-element method (FEM). ${ }^{12,13}$ A scattered-field formulation with $H_{z}=H_{z}^{s}+H_{z}^{i}$, where $H_{z}^{s}$ and $H_{z}^{i}$ are the scattered and incident fields, respectively, is used in order to reduce the dispersion error. For the incident field $H_{z}^{i}$, we consider plane wave illumination and solve the wave equation

$$
\nabla \cdot\left(\epsilon_{r}^{-1} \nabla H_{z}\right)+k_{0}^{2} \mu_{r} H_{z}=0
$$

where $\epsilon_{r}$ is the relative permittivity, $\mu_{r}$ is the relative permeability, and $k_{0}$ is the free space wave number. The computational domain is truncated on the outer boundary $\Gamma_{\mathrm{ABS}}$ using a first order absorbing boundary condition for the scattered field $\boldsymbol{n} \cdot \epsilon_{r}^{-1} \nabla H_{z}^{s}+j k_{0} \sqrt{\epsilon_{r}^{-1} \mu_{r}} H_{z}^{s}=0$, where $\boldsymbol{n}$ is the outward-pointing normal vector of the boundary and $j=\sqrt{-1}$. Equivalent equations for the $E_{z}$ polarization are easily obtained by interchanging $H_{z} \leftrightarrow E_{z}$ and $\mu_{r} \leftrightarrow \epsilon_{r}$.

We mimic a strongly scattering object by imposing a PEC boundary condition, $\boldsymbol{n} \times \boldsymbol{E}=0$, at $\Gamma_{\mathrm{PEC}}$. For the $E_{z}$ polarization, this is equivalent to $E_{z}=0$ (no field on the

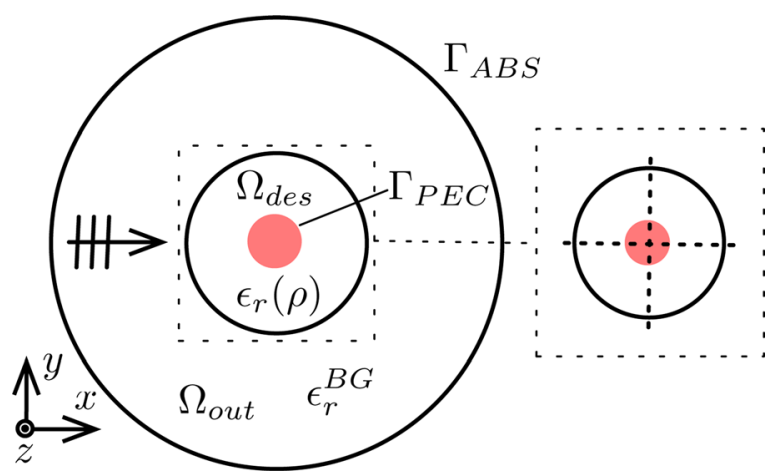

FIG. 1. (Color online) Schematics of a cylindrical cloak illuminated by either an $E_{z}$ - or an $H_{z}$-polarized uniform plane waves in a background permittivity $\epsilon_{r}^{\mathrm{BG}}$ (either vacuum or a typical dielectric). In the design domain $\Omega_{\mathrm{des}}$, the permittivity $\epsilon_{r}(\rho)$ can be varied continuously on an element basis to create the cloaking effect of a PEC cylinder (innermost region). 
boundary) while $\boldsymbol{n} \cdot\left(\epsilon_{r}^{-1} \nabla H_{z}\right)=0$ for the $H_{z}$ polarization (the field is not necessarily zero, while the radial derivative is). The latter case may support surface waves, and this difference will have an important effect on the performance of the optimized cloaks.

We employ a standard density-based topology optimization method ${ }^{11}$ and we restrict our investigations to nonmagnetic materials $\left(\mu_{r}=1\right)$. In the cloak domain wrapped around the cylinder, the relative permittivity can be varied continuously on an element basis between free space permittivity $\epsilon_{r}^{\min }=1$ and an upper bound on the permittivity $\epsilon_{r}^{\max }=2$, achievable with a broad range of naturally occurring transparent dielectric materials. For each element, we introduce a design variable, $\rho \in[0 ; 1]$, as a relative element material density. Furthermore, we make a linear interpolation of $\epsilon_{r}(\rho)$ so that

$$
\epsilon_{r}(\rho)=\epsilon_{r}^{\min }+\rho\left(\epsilon_{r}^{\max }-\epsilon_{r}^{\min }\right) .
$$

In this analysis, we test cloaks of either graded index material, which permits intermediate values of the permittivity, or discrete material designs, which do not. The graded or discrete designs are achieved by introducing a density filter ${ }^{14,15}$ or a Heaviside projection filter $^{16}$ with a robust formulation, ${ }^{17}$ respectively. The permittivity of the background material, $\epsilon_{r}^{\mathrm{BG}}$, is set to either 1 or 2 , depending on whether we are distributing dielectric material having a higher or lower permittivity than the background material, respectively. A volume constraint is imposed along with symmetry constraints on the design with two symmetry lines around which the 4 equally sized sub-domains can be mirrored as shown in Fig. 1. Utilizing the symmetry lines, all cloaks are designed to be effective for two antiparallel angles of incidence $\left(0^{\circ}\right.$ and $\left.180^{\circ}\right)$.

(a)

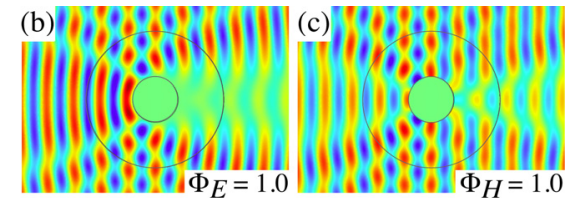

(d)
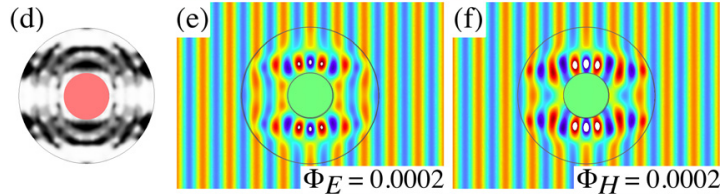

(g)
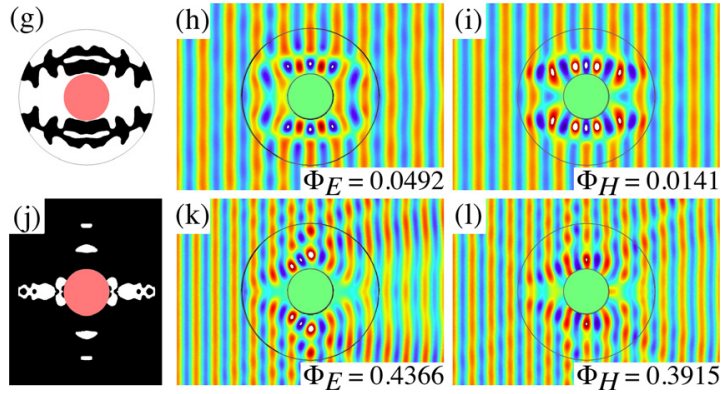

FIG. 2. (Color online) Polarization-independent cloak designs and the associated objective values $\Phi$ [Eq. (3)] for $E_{z}$ and $H_{z}$-polarized waves. The middle and rightmost panels show the total fields for $E_{z}$ and $H_{z}$ polarizations, respectively. Panels (a)-(c) show total fields for scattering of the PEC cylinder itself. Panels (d)-(f) show graded cloak designs with $\epsilon_{r}^{\mathrm{BG}}=1$, while (g)-(i) show the discrete counterparts. Likewise, panels (j)-(l) show the discrete design with $\epsilon_{r}^{\mathrm{BG}}=2$. In the linear gray-scale plots, white and black color correspond to $\epsilon_{r}=1$ and $\epsilon_{r}=2$, respectively.
In order to cloak the cylinder, the objective is to minimize the norm of the scattered field in the surroundings,

$$
\Phi=\Phi_{E}+\Phi_{H}=\frac{1}{W_{E}} \int_{\Omega_{\text {out }}}\left|E_{z}^{s}\right|^{2} \mathrm{~d} \boldsymbol{r}+\frac{1}{W_{H}} \int_{\Omega_{\mathrm{out}}}\left|H_{z}^{s}\right|^{2} \mathrm{~d} \boldsymbol{r}
$$

where $W$ is the norm of the scattered field in the outer domain when no cloak is present. This normalization makes $\Phi_{E, H}$ dimensionless and easy to interpret. If $\Phi_{E, H}<1$, cloak and cylinder produce less scattering than the non-cloaked cylinder. The design is updated iteratively using the gradient-based optimization routine method of moving asymptotes (MMA). ${ }^{18}$ The sensitivities are obtained using the adjoint method. ${ }^{19}$

Graded and discrete designs that minimize the scattering from both $E_{z}$ and $H_{z}$ polarizations simultaneously are presented in Fig. 2 (leftmost panels). The total fields for $E_{z}$ and $H_{z}$ polarizations are shown in the middle and rightmost panels, respectively. The fields from the non-cloaked cylinders for $E_{z}$ and $H_{z}$ polarizations with $\epsilon_{r}^{\mathrm{BG}}=1$ are shown in Figs. 2(b) and 2(c) as a reference. In case of $\epsilon_{r}^{\mathrm{BG}}=1$, the graded design result in scattering less than $1 \%$ of the non-cloaked cylinder and even for the more restrictive case with a discrete design scattering is reduced to less than $5 \%$ for both polarizations. The $H_{z}$ polarization for the discrete design yields less scattering than the $E_{z}$ polarization, due to the less restrictive boundary condition for the $H_{z}$ polarization. Contrary to simple intuition, considerable scattering is produced by all designs with $\epsilon_{r}^{\mathrm{BG}}=2$. Graded designs with $\epsilon_{r}^{\mathrm{BG}}=2$ produce more scattering than the discrete counter-parts; hence, we have omitted graded designs and show only the discrete design. To analyze the cause for the degeneration in cloaking performance for designs with $\epsilon_{r}^{\mathrm{BG}}=2$, we relax the problem and investigate whether or not effective cloaks with $\epsilon_{r}^{\mathrm{BG}}=2$ can be designed individually for either the $E_{z}$ or $H_{z}$ polarization.

The optimized cloaks and total fields for $E_{z}$ and $H_{z}$ polarizations are shown in Figs. 3 and 4, respectively. Even though we relax the problem and optimize individually for either one or the other polarization, both the graded and

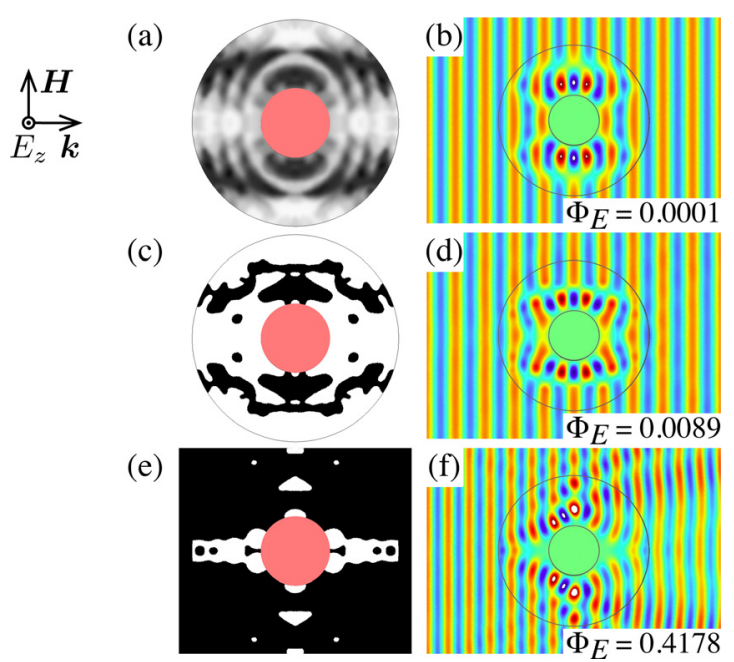

FIG. 3. (Color online) Cloak designs and associated objective values $\Phi[$ Eq. (3)] for $E_{z}$ polarization. For a description of the individual panels, see caption of Figs. 2(d)-2(1). 


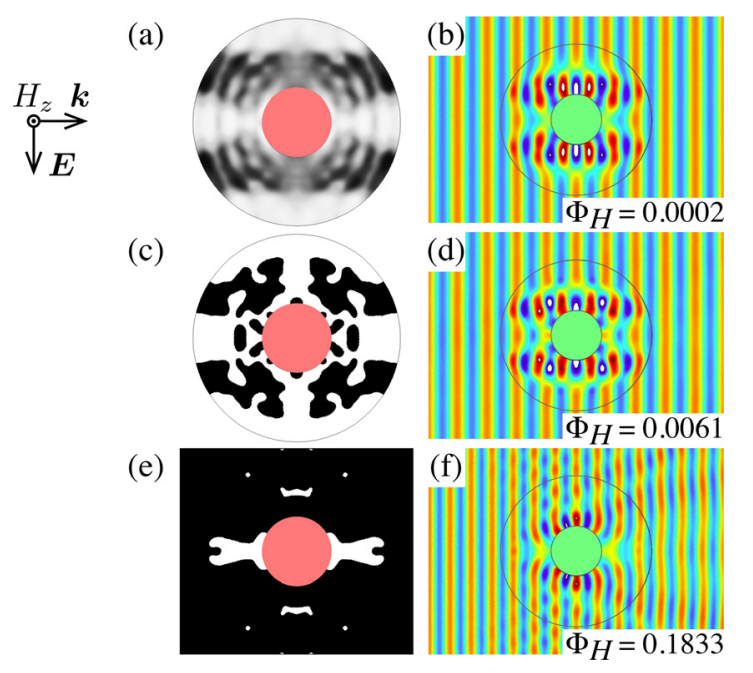

FIG. 4. (Color online) Cloak designs and associated objective values $\Phi[$ [Eq. (3)] for $H_{z}$ polarization. For a description of the individual panels, see caption of Figs. 2(d)-2(1).

discrete designs with $\epsilon_{r}^{\mathrm{BG}}=2$ result in poor cloaking performance. We thereby see that the limited cloaking performance for designs with $\epsilon_{r}^{\mathrm{BG}}=2$ is not caused by the requirement on the cloaks to be effective for both polarizations simultaneously. Instead, we conclude that it is a consequence of optical modes tending to concentrate their electric- or magnetic-field energy in regions of high dielectric values. ${ }^{20}$ For the designs with $\epsilon_{r}^{\mathrm{BG}}=1$, the field energy is concentrated in regions of higher permittivity, wherein the wave is delayed and subsequently phase matched to the waves outside the cloak. For the designs with $\epsilon_{r}^{\mathrm{BG}}=2$, we can only design regions, which the field avoids. Hence, it is possible to split the waves in front of the object with very limited backscattering. However, the waves cannot be collected on the backside of the object without scattering. For the $H_{z}$ polarization, a surface wave can exist on the PEC boundary as mentioned previously. The scattering is reduced by a factor of two for $H_{z}$ polarization with $\epsilon_{r}^{\mathrm{BG}}=2$ compared with $E_{z}$ polarization by coupling the wave into the surface mode, which makes it easier for the wave to flow around the object.

Finally, we address the radar cross section from backscattering to forward-scattering produced by either the individually optimized discrete designs or the non-cloaked cylinders (as reference), see Fig. 5. The radar cross section is normalized by the radar cross section in the backscattering direction from the non-cloaked cylinder for the $E_{z}$ polarization. For $\epsilon_{r}^{\mathrm{BG}}=2$, the backscattering of the designs are reduced significantly and are on level with the designs for $\epsilon_{r}^{\mathrm{BG}}=1$. On the contrary, the radar cross section for $\epsilon_{r}^{\mathrm{BG}}=2$ is comparable to that of the non-cloaked cylinders around the forward scattering direction.

In conclusion, we have presented directional topology optimized all-dielectric low-contrast cloaks that minimize scattering for combined $E_{z}$ and $H_{z}$ polarized waves. Nearperfect cloaking is achieved for the graded design with $\epsilon_{r}^{\mathrm{BG}}=1$ and even for a discrete design scattering for both polarizations is reduced to less than 5\% of that from the noncloaked cylinder. Furthermore, the effect of the background material has been investigated. The results indicate that fully

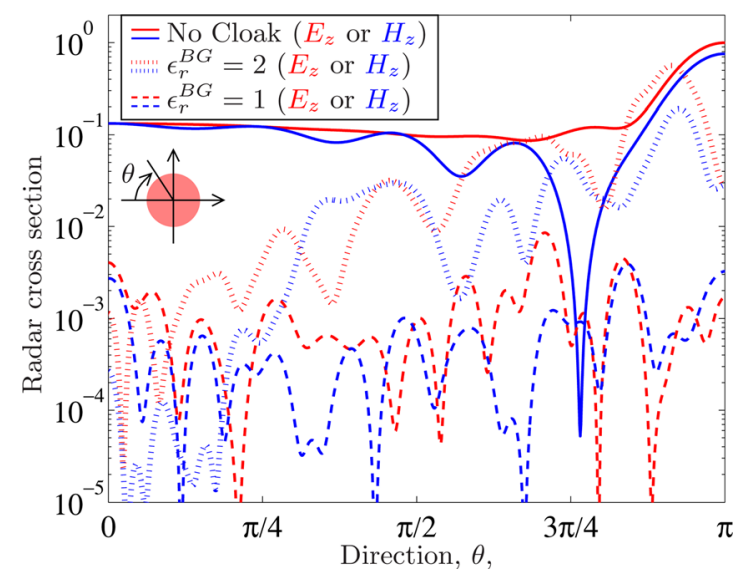

FIG. 5. (Color online) Radar cross section from back-scattering $(\theta=0)$ to the forward-scattering $(\theta=\pi)$ for the discrete designs with a background permittivity of $\epsilon_{r}^{\mathrm{BG}}=1$ (dashed lines) or $\epsilon_{r}^{\mathrm{BG}}=2$ (dotted lines) illuminated by either an $E_{z}$ or an $H_{z}$-polarized waves. Designs with $\epsilon_{r}^{\mathrm{BG}}=2$ cause limited backscattering, but with significant forward scattering. For comparison, results for the non-cloaked cylinder are also included (solid lines).

enclosed, all-dielectric, low-contrast cloaks can be designed effectively when distributing a material with higher permittivity than the background material. However, contrary to simple intuition this is not possible for the "inverse" case, i.e., when distributing a material with lower permittivity than the background material. The backscattering is reduced significantly for all optimized designs. For an $H_{z}$-polarized wave, the optimized designs can take advantage of the surface mode at the PEC boundary and achieve less scattering than the optimized designs for the $E_{z}$-polarized wave.

This work was supported by the Villum Foundation (the NextTop project) and by the Danish Research Council for Technology and Production (Grant No. 274-06-0507).

${ }^{1}$ J. B. Pendry, D. Schurig, and D. R. Smith, Science 312, 1780 (2006).

${ }^{2}$ U. Leonhardt, Science 312, 1777 (2006).

${ }^{3}$ Y. Lai, H. Y. Chen, Z.-Q. Zhang, and C. T. Chan, Phys. Rev. Lett. 102, 093901 (2009).

${ }^{4}$ D. Schurig, J. J. Mock, B. J. Justice, S. A. Cummer, J. B. Pendry, A. F. Starr, and D. R. Smith, Science 314, 977 (2006).

${ }^{5}$ J. Li and J. B. Pendry, Phys. Rev. Lett. 101, 203901 (2008).

${ }^{6}$ J. Valentine, J. Li, T. Zhentgraf, G. Bartal, and X. Zhang, Nature Mater. 8, 568 (2009).

${ }^{7}$ X. Chen, Y. Luo, J. Zhang, K. Jiang, J. B. Pendry, and S. Zhang, Nat. Commun. 2, 176 (2011).

${ }^{8}$ J. Zhang, L. Liu, Y. Luo, S. Zhang, and N. A. Mortensen, Opt. Express 19, 8625 (2011).

${ }^{9}$ T. Ergin, N. Stenger, P. Brenner, J. B. Pendry, and M. Wegener, Science 328, 337 (2010).

${ }^{10}$ J. Andkjaer and O. Sigmund, Appl. Phys. Lett. 98, 021112 (2011).

${ }^{11}$ M. P. Bendsøe and O. Sigmund, Topology Optimization: Theory, Methods and Applications (Springer, Berlin, 2003).

${ }^{12} \mathrm{~J}$. Jin, The Finite Element Method in Electromagnetics (Wiley, New York, 2002).

${ }^{13}$ COMSOL Multiphysics 3.5a, COMSOL AB, Stockholm.

${ }^{14}$ B. Bourdin, Int. J. Numer. Methods Eng. 50, 2143 (2001).

${ }^{15}$ T. E. Bruns and D. A. Tortorelli, Comput. Methods Appl. Mech. Eng. 190, 3443 (2001).

${ }^{16}$ J. K. Guest, J. H. Prévost, and T. Belytschko, Int. J. Numer. Methods Eng. 61, 238 (2004).

${ }^{17}$ F. Wang, B. S. Lazarov, and O. Sigmund, Struct. Multidiscip. Optim. 43, 767 (2011).

${ }^{18}$ K. Svandberg, Int. J. Numer. Methods Eng. 24, 359 (1987).

${ }^{19}$ J. S. Jensen and O. Sigmund, J. Opt. Soc. Am. B 22, 1191 (2005).

${ }^{20}$ J. Joannopoulos, R. Meade, and J. Winn, Photonic Crystals: Molding the Flow of Light (Princeton University, New Jersey, 1995). 